\title{
Flore de remplacement et bactériothérapie lactique en médecine vétérinaire ${ }^{(1)}$
}

\author{
par \\ M. GROS et G. THIEULIN
}

La flore du tractus digestif, selon sa nature et son importance, constitue un facteur essentiel de bonne ou de mauvaise santé.

Tel est le simple point de vue concernant l'Homme, et aussi les précieux animaux de compagnie qui interviennent, parfois si fortement, dans notre vie affective.

Pour ceux de nos animaux domestiques dont l'exploitation est strictement d'ordre économique, cette flore influence de façon plus ou moins intense le rendement de leur élevage puisque se trouvent sous sa dépendance la croissance des jeunes et l'équilibre organique des adultes.

Très complexe, inégale et variée, la flore intestinale présente pour chaque espèce, à l'état normal, à la fois une composition spécifique et une certaine stabilité. Son maintien et sa stimulation règlent et favorisent les processus physiologiques de la digestion et de l'assimilation, d'où tout découle, directement ou indirectement.

\section{I. - NATURE ET IMPORTANCE}

Le liquide de rumen compte environ $10^{9}$ germes par ml et, mêlée à cet ensemble, une gamme de protozoaires très variés évaluée à $10^{6}$ par g de contenu de la panse.

La population bactérienne varie selon l'alimentation : elle diminue lorsque la ration compte des aliments de mauvaise qualité, les grains amènent une prédominance de germes anaérobies facultatifs.

Cependant, certains auteurs (Gal, Annisson et coll.) estiment qu'elle comporte un " noyau » relativement stable. C'est ainsi qu'une vingtaine de types de bactéries existerait de façon constante

(1) Recueil de Médecine vétérinaire, 1968, n 9, avec complément p. 150. 
et que 85 à 90 p. 100 des micro-organismes isolés appartiendraient à l'un de ces types. Par ailleurs, les mêmes espèces microbiennes existeraient dans le rumen des bovins élevés dans des régions différentes mais soumis à la même alimentation. Ajoutons que nombre de ces germes sont cellulolytiques, certains étant en même temps amylolytiques.

Il est admis que les éléments les plus caractéristiques appartiennent aux familles suivantes :

- Streptocoques (notamment Streptococcus bovis), dont le nombre augmente après administration de glucose et d'amidon.

- Bactéries cellulolytiques (anaérobies stricts), dont : Bactéroïdes succinogènes, non sporulés - Ruminobacter parvum - Ruminococcus flavefaciens.

- Lactobacilles (dont le nombre peut dépasser $10^{6}$ par $\mathrm{ml}$ ), surtout abondants, d'une part, chez les jeunes veaux et, d'autre part, lorsque le régime comprend beaucoup de foin et des fourrages concentrés.

Précisons que les principaux produits du métabolisme sont l'acide succinique, l'acide acétique, l'acide formique, l'acide butyrique, $\mathrm{H}_{2}$ et $\mathrm{CO}_{2}$.

Les protozoaires ne semblent pas faire partie du noyau de base de la population du rumen; ils disparaissent avec le jeûne.

Dans le cæcum du bœuf, dans son intestin, on décèle une flore voisine de celle du rumen, les mêmes variations liées à l'alimentation s'y retrouvent avec une prédominance des fermentations basiques, et des putréfactions en cas d'alimentation protidique.

La distinction des bactéries iodophiles ne semble pas significative. Selon Demole, ces bactéries n'appartiennent pas à une race ou à une espèce particulière. La transformation iodophile des bactéries serait consécutive à un phénomène digestif, le plus souvent normal, caractérisé par des fermentations, et l'iodophilie est le témoin mais non pas la cause de ces fermentations exagérées.

La flore digestive du jeune veau avant sevrage est naturellement très différente. Cependant, on trouve dans le rumen comme dans la caillette, une très grande abondance de lactobacilles.

Dans l'intestin, les coliformes dominent. Smith et Crabb ont isolé de l'intestin de veau sain plusieurs dizaines de types d'Escherichia coli.

Chez le mouton, l'appareil digestif ne présente pas de différences telles qu'il puisse en résulter une modification appréciable de la flore. Le rumen entretient une population analogue, y compris les protozoaires.

Chez le cheval, l'estomac est pratiquement stérile en raison de la forte acidité du suc gastrique. Dans le cæcum, on trouve une flore de fermentation avec également la présence de protozoaires. 
Chez le porc, l'estomac est le siège d'un abondant développement microbien : le fait est assez particulier. Le tube digestif contient toute une série de micro-organismes. Déjà dans la bouche, on trouve des entérocoques, des staphylocoques, des amibes. La portion terminale de l'intestin grêle contient surtout des bactéries parmi lesquelles Escherichia coli domine.

Chez le chien, l'estomac et la partie proximale de l'intestin grêle sont pratiquement stériles. On n'isole jamais de staphylocoques de l'intestin mais on y trouve Escherichia coli, des entérocoques et Clostridium perfringens. Ce dernier germe est influencé par le régime alimentaire : il diminue en période de jeûne et est favorisé dans son développement par le régime carné.

Chez le lapin, où la coprophagie est de règle, la flore microbienne, selon Berrens, compte un grand nombre de staphylocoques.

Chez le cobaye enfin, Berrens estime que 80 p. 100 de la flore est constituée par des lactobacilles acidophiles et que les coliformes et les entérocoques sont assez rares.

Notons que, si la vie aseptique est possible dans certaines espèces, et sous diverses conditions, une flore intestinale semble indispensable tout au moins chez les ruminants. Du rapide examen qui vient d'être fait, on voit, en effet, que leur nutrition est assurée en grande partie par les micro-organismes de leurs réservoirs digestifs, toute une flore microbienne contribuant à la dégradation de la cellulose.

Dans les autres espèces, une flore intestinale relativement stable et constante conditionne un équilibre biologique propre aux sujets sains.

\section{II. - MODIFICATIONS DE LA FLORE}

Trois causes essentielles sont à la base des modifications qualitatives et quantitatives de la micro-population du tractus digestif :

a) Etats pathologiques.

b) Variations du régime alimentaire.

c) Actions thérapeutiques.

a) Sous l'influence d'infections microbiennes ou parasitaires, on peut noter :

- une invasion ascendante vers les segments digestifs normalement stériles ;

- un déséquilibre, par excès, d'éléments microbiens habituellement présents prenant alors, soit des formes anormales, soit un pouvoir pathogène plus ou moins accentué.

Ainsi le colibacille peut devenir soudain responsable d'entérites très graves et de septicémies. 
- Migration de germes hors de l'intestin dans la circulation générale (colonisation fréquente des voies urinaires).

- Libération excessive d'ammoniaque et de bases aromatiques au cours de la digestion des protides par les germes de putréfaction.

- Multiplication et exaltation de la virulence de germes normalement rares ou peu actifs.

b) L'alimentation glucidique favorise les fermentations acides alors que l'alimentation protidique entraîne des fermentations basiques et des putréfactions. Le remplacement du fourrage de prairies pauvres par du fourrage de bonne qualité fait apparaître dans le rumen des bactéries iodophiles, puis des levures.

Les aliments grossiers ou de digestion difficile provoquent une irritation de la paroi intestinale amenant une modification profonde de la flore.

En dehors de l'influence des constituants de la ration, les aliments peuvent apporter de nombreux micro-organismes (ferments lactiques, ferments butyriques, bactéries cellulolytiques, des colibacilles, des moisissures...) : tel est le cas notamment des ensilages.

Soulignons l'importance de $W$. perfringens dont le type A en particulier, hôte habituel des voies digestives, est capable sous certaines influences (régime trop riche en protides, surcharge alimentaire, changement brusque de l'alimentation...) de provoquer de redoutables entérotoxémies.

Une alcalinisation du milieu favorise la multiplication de ce germe anaérobie très répandu pour aboutir à la production de sa toxine qui va diffuser dans l'organisme jusqu'aux lieux d'élection.

c) Les études concernant l'incidence des médications antidiarrhéiques et antiparasitaires sont rares mais les heureux effets des réensemencements des voies digestives en ferments lactiques chez le veau, après traitements anticoccidiens, apportent la preuve qu'effectivement les médicaments utilisés avaient déséquilibré la population microbienne.

Par contre, les accidents intestinaux de l'antibiothérapie ont été étudiés de façon approfondie en médecine humaine : anites ou anorectites - diarrhées simples et syndromes cholériformes - surinfections par Candida albicans.

\section{III. - RENFORCEMENT OU RECONSTITUTION DE LA FLORE NORMALE}

Le fait est là : les conséquences fâcheuses des perturbations de la flore digestive tout autant que celles, heureuses, du retour à son équilibre normal, ont été depuis longtemps notées. 
La théorie de Metchnikoff sur la cause des effets bienfaisants, en médecine humaine, dus à l'ingestion de produits à base de ferments lactiques - tels le yaourt, le kéfir et autres laits fermentés s'est avérée fausse : il n'est plus admis que le bacille bulgare et les streptocoques lactiques puissent proliférer dans le milieu intestinal.

Cependant, on ne peut nier les résultats obtenus par la bactériothérapie lactique.

Les analyses de laboratoire ont, chez l'homme, permis de suivre l'évolution des modifications de la flore intestinale en rapport avec les troubles ressentis et les désordres constatés, puis avec le retour à l'état physiologique.

Chez nos animaux domestiques, les mêmes analyses sont, dès les prélèvements à effectuer, d'une grande complexité, mais de très nombreuses observations cliniques ont apporté, dans le même sens, un témoignage valable.

\section{a) Préparations biologiques utilisées}

Ces préparations se répartissent en deux groupes : les préparations à action indirecte et les préparations à action directe.

Les premières mettent en jeu des micro-organismes n'appartenant pas à la flore normale ou des éléments de cette flore tués ou présentés sous forme de filtrats.

Dans ce groupe, figurent les levures, le bacille subtil, les lysats colibacillaires, lactiques ou polymicrobiens..., qui semblent agir en favorisant le développement de la flore présente, même réduite. L'action obtenue serait engendrée par les produits du métabolisme microbien riches en facteurs de croissance (acides aminés, enzymes...).

Les préparations à action directe font intervenir les hôtes habituels de l'intestin et du rumen. Il s'agit avant tout des lactobacilles, anaérobies facultatifs, produisant de l'acide lactique à partir des glucides.

Parmi eux, Lactobacillus acidophilus est généralement préféré en raison de sa réputation à pouvoir se multiplier in situ. Bifidobacterium bifidum, élément important de la flore chez le nourrisson est recherché en médecine infantile, mais son inconstance peut le faire considérer comme une arme à double tranchant. Escherichia coli est utilisé également vivant, mais le choix de la souche est assez délicat, car la démarcation entre colibacilles pathogènes, non pathogènes et ceux susceptibles de devenir dangereux, est, dans de nombreux cas, impossible à fixer.

Outre ces différentes préparations, citons celles à base de bactériophages colibacillaires et toutes celles qui associent des germes tués et des germes vivants, des levures, et, d'une façon générale, de diverses associations pouvant présenter un intérêt. 
Des combinaisons de souches cellulolytiques sont plus spécialement à considérer pour l'ensemencement du rumen.

Enfin, signalons l'adjonction de levures, de ferments lactiques, et d'enzymes à des antibiotiques.

\section{b) Conditions d'efficacité}

Bien que certaines préparations de germes tués aient donné de bons résultats, à la fois semble-t-il, par l'effet de produits de métabolisme des cultures - facteurs de croissance risquant d'ailleurs de favoriser des microbes indésirables - et par celui de l'excipient lui-même, l'importance de micro-organismes vivants, c'est-à-dire permettant un réensemencement, recueille, à bon droit, la faveur générale.

Autres conditions d'efficacité :

- Vitalité des germes ;

- Administration de doses importantes ;

- Pénétration des germes dans le milieu intestinal ;

- Résistance aux antibiotiques.

La conservation de la vitalité des germes jusqu'au moment de leur utilisation constitue une nécessité évidente. Ces germes sont fragiles et leur culture s'anémie assez rapidement. Des échecs ont été souvent dus au fait qu'au moment de leur administration, les préparations ne contenaient plus qu'une flore très affaiblie ou même devenue pratiquement nulle.

La lyophilisation a résolu le problème en permettant une longue stabilisation. Le conditionnement sous vide ou sous azote apporte un complément de sécurité : l'atténuation qui se produit fatalement avec le temps demeure faible.

En partant d'une densité élevée, les résultats seront très bons.

Ainsi, l'ingestion, sous un petit volume, de doses importantes de germes dont la vitalité peut être maintenue pendant plusieurs mois devient couramment réalisable.

Par ailleurs, les cultures à forte densité microbienne, placées en excipient convenable, bien conservées comme il vient d'être dit, doivent pouvoir franchir la barrière gastrique.

$\mathrm{Si}$, chez le nouveau-né, la flore se développe dès les premiers repas, si comme nous le disions précédemment, les observations cliniques apportent des témoignages quant à la pénétration dans l'intestin de la flore de remplacement, les expérimentations conduites ces dernières années par Carraz et Moulin ont prouvé la survie de Lactobacillus acidophilus dans divers milieux biologiques (suc gastrique, bile, liquide pancréatique, selles). Une telle étude réalisée chez l'homme possède une signification générale. 
Ces auteurs ont conclu que Lactobacillus acidophilus lyophilisé conservait sa vitalité au moins deux jours à $37^{\circ} \mathrm{C}$ dans les produits ci-dessus énumérés.

La résistance à l'acidité gastrique n'est pas pour nous surprendre puisque l'une des meilleures méthodes pour l'isolement du germe, ici considéré, consiste à le placer pendant $24 \mathrm{~h}$ dans un milieu à $\mathrm{pH} \mathrm{3,5.}$

Cette caractéristique est, du reste, propre à toute une série de germes producteurs d'acide lactique.

Enfin : résistance aux antibiotiques.

Les antibiotiques étant à l'heure actuelle utilisés massivement, les micro-organismes en cause doivent pouvoir se multiplier en leur présence.

Les flores de remplacement, à base de ferments lactiques, sont à prescrire pour éviter les accidents consécutifs à l'antibiothérapie : les germes introduits vont donc se trouver en présence, pour le moins, de traces d'antibiotiques qui ne doivent pas gêner leur développement.

Il n'est pas conseillé de vouloir conférer à une souche déterminée une résistance à l'égard de plusieurs antibiotiques, mieux vaut réunir en une même préparation, plusieurs souches mono-résistantes.

La résistance des flores de remplacement à l'égard de sulfamides mériterait, pensons-nous, d'être étudiée. Il faudrait également penser à les protéger contre les antibiotiques injectables dont l'action sur la flore digestive n'est pas négligeable.

Un mot sur le choix des germes à utiliser et sur les facteurs favorables à leur implantation.

Des travaux effectués et des observations faites, il nous paraît que, si la bactériothérapie lactique doit être à base de germes vivants lyophilisée, la préférence doit être donnée dans le vaste groupe en question aux lactobacilles et notamment à Lactobacillus acidophilus.

Quant aux facteurs favorables à rechercher dans la composition du milieu intestinal pour favoriser les réensemencements, il convient de noter, selon l'âge et les espèces animales, les aliments suivants :

- le fourrage de prairies naturelles et la paille ;

- les céréales pauvres en protides et leurs issues ;

- les racines;

- le lait.

Sont à déconseiller : les fourrages de prairies artificielles, les tourteaux et les céréales.

Ajoutons que l'on conseillera utilement l'administration simultanée de lactose, d'extraits de levures, de dextrine, de lait écrémé, d'acide lactique ou d'acide citrique et de vitamines. 


\section{c) Mode d'action}

On explique habituellement comme suit l'action favorable de la bactériothérapie lactique :

1) Enrichissement et stimulation de la flore lactique locale : les conditions énoncées ci-dessus étant remplies, c'est-à-dire, germes introduits adaptés au milieu intestinal, vivants et vigoureux, antibiorésistants, absorbés en grande quantité, il y a alors enrichissement direct de la flore lactique intestinale.

Les éléments ayant résisté au suc gastrique et à la bile cultivent directement. Les éléments n'ayant pas résisté n'en apportent pas moins divers facteurs de croissance sous forme du ferment jaune de Warburg et d'une bivalence $\mathrm{B}_{1}-\mathrm{B}_{2}$.

2) Phénomènes d'antagonisme: Une action antagoniste résulte de la production de l'acide lactique qui va inhiber plus ou moins fortement le développement et le métabolisme des bactéries mal adaptées à un $\mathrm{pH}$ bas, en particulier, les éléments de la flore de putréfaction.

3) Apports vitaminiques : Les bactéries intestinales synthétisent diverses vitamines. Les perturbations de la flore normale peuvent aboutir à des carences que la flore de remplacement est à même de compenser directement ou indirectement.

4) Action antitoxique : La flore lactique renforce la protection de l'épithélium.

Une action antihistaminique certaine a été constatée que certains auteurs expliquent par l'élimination ou la neutralisation des microorganismes histaminogènes.

Enfin, la richesse de la flore en lactobacilles augmente le seuil de résistance organique à l'endotoxine colibacillaire.

\section{IV. - ESSAIS CLINIQUES}

Une expérimentation déjà longue que nous ne pouvons citer ici que partiellement a montré l'intérêt de la bactériothérapie lactique en médecine vétérinaire. Les moyens techniques chaque jour perfectionnés et notamment la lyophilisation ont, sans cesse, amélioré les résultats obtenus.

En 1926, l'emploi de cultures sèches de ferments lactiques, à doses massives, apportait à Pollalis des résultats très favorables dans le traitement des gastro-entérites et des affections cutanées (en particulier l'eczéma) du chien et du chat. De même, chez les équidés, dans la thérapeutique de lésions cutanées rebelles, notamment de l'extrémité des membres, et encore contre des affections du tractus génital chez la chienne. 
Cantenot, en 1945, rappelait les recherches de Doldi en Italie et de M. Faguet en France, sur l'action in vitro des ferments lactiques, montrant que ces germes agissent autrement que par leur seule multiplication ou leur acidité sur les microbes pathogènes, et il concluait ses propres expérimentations en ces termes : l'emploi de ferments lactiques tyndallisés s'est affirmé favorable dans les affections gastro-intestinales, particulièrement chez les jeunes, dans les maladies de la peau de nature diathésique (eczéma, urticaire...), dans le traitement des plaies, brûlures, affections de l'appareil génital.

En 1952, Ozcan relatait la valeur, dans les infections intestinales du veau (diarrhées), de l'administration de ferments lactiques vivants par voie buccale. Il s'agissait de Lactobacillus acidophilus à 98 p. 100 et, pour le reste, de streptocoques lactiques.

En 1955, Mouillard déclarait : « Une technique moderne de conservation des ferments lactiques vivants, la lyophilisation, a permis de remettre en question le principe de la bactériothérapie lactique...

Là où les sérums, vaccins, antidiarrhétiques chimiques, sulfamides et même antibiotiques échouent, les ferments lactiques lyophilisés peuvent rendre les plus grands services.

Le pronostic des entérites accompagnant les formes septicémiques conserve toute sa gravité et il est alors nécessaire d'avoir recours aux antibiotiques. Mais là, encore, les ferments lactiques seront utilisés pendant la période de convalescence dans le but de fournir au milieu intestinal des micro-organismes utiles et d'éviter le retour d'une flore pathogène.

Il est donc temps que la bactériothérapie lactique prennent l'ampleur qu'elle mérite ».

Plus récemment, en 1965, Vigneron a utilisé un mélange de Lactobacillus acidophilus et d'Escherichia coli non pathogène. Il a choisi comme terrain d'épreuve des exploitations où les veaux étaient régulièrement atteints de formes intestinales graves.

Sur 234 veaux traités dès la naissance, 142 n'ont jamais présenté de diarrhée. Sur les 92 autres malades, 60 se trouvaient dans deux élevages où la septicémie sévissait gravement avant l'administration du produit.

Cet auteur fait, par ailleurs, quelques réserves sur l'opportunité d'utiliser $E$. coli en thérapeutique ; à son avis, ce germe, bien qu'il entre dans la composition de la flore normale, peut reprendre son pouvoir pathogène.

Par ailleurs, nous avons personnellement suivi quelques emplois de Lactobacillus acidophilus seul ou associé au streptocoque ther- 
mophile, tous deux antibiorésistants, dans un mélange enrichi en vitamines $A, D_{3}, E$ et $C$. Les résultats ont été les suivants :

- Prévention de la septicémie des veaux : résultats positifs dans l'ensemble avec une plus grande efficacité lorsque l'administration est importante dès la naissance, et répétée.

- Traitement des troubles digestifs des bovins : les troubles digestifs, d'origine alimentaire et nutritionnelle, sont manifestement justiciables de la bactériothérapie lactique qui semble même être le traitement de choix des diarrhées laiteuses (dues à un changement de régime par passage du sec au vert), des diarrhées dues à une alimentation irrationnelle en période de sevrage, d'entérites hémorragiques (en association avec antiseptiques et antihémorragiques), et des troubles de l'alimentation.

- Correction de l'antibiothérapie : les veaux traités par antibiotiques et qui reçoivent ensuite des ferments lactiques se rétablissent comparativement mieux que des témoins et ont une reprise de poids plus rapide.

Le même phénomène a été observé après traitement anticoccidien : convalescence plus courte.

Tissandier, en thérapeutique porcine, a soigneusement expérimenté, en 1965 et 1966, une préparation dans laquelle entrent, outre Lactobacillus acidophilus, des vitamines et du gluconate de fer. Les résultats ont été remarquables, spectaculaires même, sur des portées de porcelets diarrhéiques et déficients : reprise rapide de l'appétit, cessation des troubles digestifs, allure générale rapidement excellente. L'amélioration était déjà sensible dès la première ingestion.

Le complexe "Lactobacillus acidophilus - sel de fer " amène non seulement la guérison des diarrhées du sevrage, mais améliore rapidement l'état général des animaux. Peut-être s'agit-il d'une meilleure assimilation du fer en présence des ferments lactiques ?

Enfin, Robert E. Hungate, effectuant une étude générale sur l'emploi bénéfique des ferments lactiques et, notamment, de Lactobacillus acidophilus, en thérapeutique vétérinaire, souligne le grand intérêt d'un tel emploi dans les cas suivants :

- Manque d'appétit et troubles de fonctionnement de l'appareil digestif, par insuffisances ou inversions de la flore normale, naturelles ou consécutives à un traitement, principalement lorsqu'il s'agit d'antibiothérapie.

- Périodes de transition dans le régime alimentaire, principalement chez le veau, portant nécessité d'une adaptation.

- Fermentations anormales.

- Indigestions.

- Certaines intoxications.

- Affections, locales ou générales, des nouveau-nés.

Et cet Auteur conclut en recommandant l'addition de vitamines $\mathrm{A}$ et $\mathrm{D}$ aux préparations de ferments lactiques administrées. 


\section{CONCLUSION}

De nombreuses questions posées du point de vue scientifique sur le problème des flores de remplacement n'ont pas encore reçu de réponse. Des progrès dans nos connaissances à ce sujet peuvent être attendus de la pyxigraphie adaptée aussi bien pour l'étude des flores normales que pour les modalités d'administration. La technique d'immuno-fluorescence est également susceptible d'apporter d'intéressantes précisions sur le sort des micro-organismes absorbés.

A l'heure actuelle, les résultats cliniques ont confirmé dans l'ensemble les hypothèses généralement admises sur l'implantation et l'efficacité de la flore lactique.

Les moyens techniques dont nous disposons maintenant pour réaliser les préparations désirées et leur conservation, mis au service d'un choix approprié des formules globales d'administration, conditionnent l'efficacité des produits présentés.

La posologie ainsi que les règles d'alimentation à suivre en cours de traitement sont également à préciser.

Parmi les germes à retenir, Lactobacillus acidophilus, particulièrement bien connu, d'une innocuité absolue et d'une valeur indiscutable, demeure l'élément essentiel.

Les effets de l'arsenal thérapeutique puissant dont disposent les cliniciens vont souvent trop loin, et des correcteurs biologiques sont alors les bienvenus.

Si l'emploi des antiseptiques de tous ordres, antibiotiques et pesticides, ne peut qu'être admis comme une nécessité médicale et économique nous pensons que le biologiste a le devoir de protéger les organismes qui lui sont confiés contre les conséquences, parfois redoutables, et les abus, de certaines thérapeutiques ou pratiques alimentaires modernes.

Action préventive et action curative, simple ou associée : telle est la double modalité d'intervention que, sous les conditions d'activité énoncées, le praticien vétérinaire et l'éleveur peuvent solliciter de l'emploi judicieux des ferments lactiques.

\section{Références bibliographiques}

ALARçon (J.L.) (1961). - Les accidents intestinaux consécutifs à l'emploi d'antibiotiques et leur traitement. Semana Médica di Mexico, Volume xxix, $\mathrm{n}^{\circ} 8,307$.

Annison (E. F.) et Lewis (D.) (1959). - Metabolism in the Rumen. Methuen and Co. Ed., Londres.

Bargheon (K.) (1965). - Dysmicrobismes intestinaux. Revue Française de Gastro-Entérologie, $\mathrm{n}^{\circ} 11$. 
BECK (C.) et Necheles (H.) (1961). - Beneficial effects of administration of Lactobacillus acidophilus in diarrheal and other intestinal disorders. American Journal of gastro-enterology, 35/522.

Belonovsky (1921). - Influence du ferment lactique sur la flore des excréments de souris. Annales de l'Institut Pasteur, 991.

BERRENS (H.) (1965). - Flore intestinale normale et diététique. Journée nationale de diététique, Paris.

Boouien (Y.) (1955). - Troubles digestifs provoqués par les antibiotiques. Archives des maladies de l'appareil digestif et des maladies de nutrition, suppl. au $\mathrm{n}^{\circ}$ 6, 132.

Busson (A.) et Davezac (J.F.) (1951). - Les diarrhées subaiguës et chroniques post-antibiotiques. Entretiens de Bichat (Médecine).

Buttiaux (R.), Tacquet (A.) et Gaudier (B.) (1955). - Bactériologie des syndromes intestinaux provoqués par les antibiotiques. Journées des gastroentérologues français, Paris.

Cantenot (G.) (1946). - Contribution à l'étude des ferments lactiques tyndallisés : leur emploi en thérapeutique vétérinaire. Thèse Doctorat Vétérinaire, Lyon.

Carraz (M.) et Moulin (A.) (1958). - Etude de la survie de Lact, acidophilus dans divers milieux biologiques. Communication à la Société de Biologie, Filiale de Lyon.

Carraz (M.) et Moulin (A.) (1958). - Etude du transit chez l'homme de l'acidophilus résistant aux antibiotiques. Communication à la Société de Biologie, Filiale de Lyon.

Cattan (R.) et Milstein (J.) (1960). - La thérapeutique lactique dans les affections coliques. Presse Médicale, 68, 25.

Christol (D.) (1965). - Les antibiotiques et les flores de remplacement. Gazette Médicale de France, $\mathrm{n}^{\circ} 10$.

Demole (M.) (1965). - Quelques aspects cliniques des déviations de la flore intestinale. Journée nationale de Diététique. Documentation technique pharmaceutique, 175, rue du Faubourg-Poisonnière, Paris $\left(9^{e}\right)$.

Desvignes (1965), - Flore de remplacement et Diététique. Microbiologie et Diététique, 91.

DuchesNAY (G.) (1953). - Les accidents thérapeutiques provoqués par les antibiotiques d'origine fungique. Thérapie, 4, 632.

GALL et coll (1958). - in « Données générales sur la Nutrition et l'Alimentation " (Nouvelle Encyclopédie agricole, Vol. 1) par JAcouor, LE BARS et Simonnet, J. B. Baillière Ed., Paris, p. 210.

Hungate (Robert E.) (1966). - The rumen and its microbes. Academic Press, Londres W. 1, Berkeley, square House.

Kaffarnik (H.), Husmann (F.), Schmid (W.) et Junchens (R.) (1966. Comportement de la flore intestinale de l'homme sous l'effet qu'exerce l'administration d'antibiotiques et de lactobactéries lyophilisées. Gostroentérologia, 106, 501, 511.

KleinKauf (I.) (1959). - Expériences sur l'utilisation de souches acidophiles résistants durant la thérapeutique antibiotique. Kinderheilkunde, tome 160 , $\mathrm{n}^{\circ} 1,51$.

Kopeloff (N.) (1923). - L'action du Bacillus acidophilus est-elle un phénomène strictement bactériologique? Journal American medical association, $\mathrm{n}^{\circ} 9,602$.

Lasserre (R.), Gaschen (M.) et Demole (M.) (1965). - Essai d'implantation de bacilles lactiques dans le côlon par pyxigraphie. Gastro-enterologia, $103,171$. 
LEDuc (M.) (1951). - Contribution à l'étude des ferments lactiques vivants : leur emploi en médecine vétérinaire. Thèse Doctorat vétérinaire, Alfort, Paris.

Luccio (J. J.) (1961). - Expérimentation clinique d'une nouvelle souche de Lacto-acidophilus dans les affections gastro-intestinales. Thèse de Doctorat en Médecine, Mexico.

Metchnikoff (1909). - Traité de thérapeutique, Paris. Annales Institut Pasteur 1910-45, 1912-26, 1913-27.

MouillaRd (1955). - Emploi des ferments lactiques lyophilisés dans le traitement de l'entérite des veaux nouveau-nés. Thèse de Doctorat Vétérinaire, Alfort, Paris.

Ozcan (C.) (1952). - Maladie des nouveau-nés : traitement de la diarrhée des veaux par les ferments lactiques. Thèse Doctorat Vétérinaire, Alfort, Paris.

Pollalis (P.) (1926). - Les ferments lactiques et leurs applications en médecine vétérinaire. Thèse Doctorat Vétérinaire, Alfort, Paris.

Smith (H.W.) et CRABB (W. E.) (1901). - The bacterial flora of the faeces of animals and man. Its development in the young. Journal Path. Bact., $82,53$.

Tissandier (Henri) (1968). - Bactériothérapie lactique, notamment chez le porcelet. Thèse Doctorat Vétérinaire, Alfort, Paris.

Vigneron (P. M.) (1965). - Quelques usages de ferments lactiques lyophilisés en médecine vétérinaire. Thèse de Doctorat Vétérinaire, Toulouse.

Warburg (O.) et Christian (W.) (1933). - Bioch. Zeitsch, 260, 489.

Winkelstein (A.) (1956). - Lactobacillus acidophilus tablets in the therapy of functional intestinal disorders. American Practitioner and digest of treatment. Philadelphia Vol. 7, $\mathrm{n}^{\circ} 10,1-637$. 